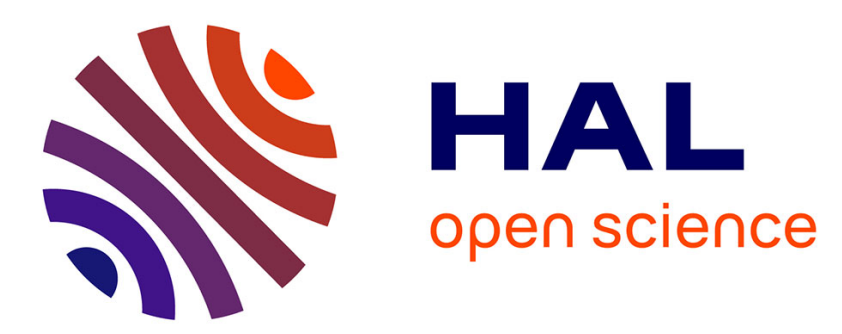

\title{
La quête des températures ultrabasses
}

Daniel Suchet

\section{To cite this version:}

Daniel Suchet. La quête des températures ultrabasses. Daniel Chartier and Jan Borm. Le Froid. Adaptation, production, représentations, effets., Les Presses de l'Université du Québec, A paraître. hal-01675654

\section{HAL Id: hal-01675654 https://hal.science/hal-01675654}

Submitted on 16 Jan 2018

HAL is a multi-disciplinary open access archive for the deposit and dissemination of scientific research documents, whether they are published or not. The documents may come from teaching and research institutions in France or abroad, or from public or private research centers.
L'archive ouverte pluridisciplinaire HAL, est destinée au dépôt et à la diffusion de documents scientifiques de niveau recherche, publiés ou non, émanant des établissements d'enseignement et de recherche français ou étrangers, des laboratoires publics ou privés. 


\title{
La quête des températures ultrabasses
}

\author{
Daniel Suchet
}

14 Mars 2014

\author{
Laboratoire Kastler Brossel, ENS-PSL Research University, CNRS, UPMC, Collège de France, 24 rue \\ Lhomond, 75005 Paris, France \\ daniel.suchet@polytechnique.org
}

\begin{abstract}
Résumé
Des vendeurs de glace à l'industrie cryogénique, le froid est souvent perçu comme un produit. Pour le physicien, le froid, réduction de l'agitation aléatoire des particules, apparait plutôt comme une force productrice, capable de faire émerger des structures complexes et de métamorphoser les propriétés des objets. Les basses températures ont été l'enjeu d'une course effrénée dont la première phase a pris fin en 1908 avec la liquéfaction de l'ensemble des gaz connus. Loin de clore l'aventure, cette étape a fait surgir des nouveaux états de la matière qui ne peuvent être interprétés que par la mécanique quantique. Pour étudier la supraconductivité, la superfluidité ou la condensation de Bose-Einstein, les expériences actuelles visent des températures dites ultrabasses, à quelques milliardièmes de degré au-dessus du zéro absolu. Cette présentation propose une mise en perspective historique de la recherche des très basses températures et une introduction aux expériences actuelles d'atomes froids.
\end{abstract}

Le froid change le monde autour de nous. Il transforme l'eau en glace, la pluie en neige et notre souffle en brouillard. Mais ces observations quotidiennes ne rendent compte que d'une infime partie des modifications engendrées par le froid. À de très basses températures, des phénomènes nouveaux apparaissent et le monde change du tout au tout. Dès le XVIIIe siècle, les scientifiques tentent d'imaginer les conséquences d'une chute brutale de la température. Ainsi, en 1789, Antoine Laurent de Lavoisier imagine que, tout comme la vapeur d'eau peut se condenser en gouttelettes, l'air pourrait devenir liquide s'il était soumis à un froid suffisamment intense 1 :

Si la terre se trouvait tout à coup placée dans des régions très froides, l'eau qui forme aujourd'hui nos fleuves et nos mers, et probablement le plus grand nombre des fluides que nous connaissons, se transformerait en montagnes solides. [...] L'air, dans cette supposition, ou au moins une partie des substances aériformes qui le composent, cesseraient sans doute d'exister dans l'état de vapeurs [...], faute d'un degré de chaleur suffisant; elles reviendraient donc à l'état de liquidité, et il en résulterait de nouveaux liquides dont nous n'avons aucune idée.

Initiée par Lavoisier et ses contemporains, la quête du froid cherche à atteindre des températures de plus en plus basses et à étudier les propriétés de la matière soumise à ces conditions extrêmes. Si le domaine de recherche a connu de nombreuses révolutions, il reste particulièrement actif à l'heure actuelle et met en évidence l'importance de la mécanique quantique.

En plongeant vers des températures de plus en plus basses, on pourra retracer les grandes étapes historiques de la quête du froid et présenter les propriétés surprenantes qui surgissent lorsque le froid devient prédominant. Comme toute plongée, cette exploration se fera par paliers successifs : nous allons d'abord descendre jusqu'à $-269^{\circ} \mathrm{C}$, en regardant comment les éléments peuvent passer d'un état gazeux à un état liquide, puis solide. Un palier intermédiaire jusqu'à $-273^{\circ} \mathrm{C}$ permettra de faire apparaître de nouveaux changements

1. Antoine Laurent de Lavoisier, Traité élémentaire de Chimie, Paris, Cuchet, 1789, p. 33. 
d'état, moins familiers, tels que la transition vers la supraconductivité. Enfin, on pourra s'aventurer au plus près du zéro absolu en abordant le thème des atomes ultrafroids.

Tout au long de cette exploration des basses températures, on verra comment le froid, en réduisant l'agitation chaotique des particules, permet l'apparition de structures de plus en plus complexes.

\section{De $20^{\circ} \mathrm{C}$ à $-269^{\circ} \mathrm{C}$. Solide, liquide ou gaz?}

En 1789, la proposition de Lavoisier est extrêmement spéculative : ce n'est que par analogie avec les quelques gaz connus à l'époque, et en particulier la vapeur d'eau, que Lavoisier formule son idée. Pour comprendre les mécanismes à l'oeuvre derrière la liquéfaction de l'eau et estimer leur capacité à liquéfier d'autres gaz, il nous faut nous entendre sur ce qu'est la température.

Depuis l'Antiquité, la question a longtemps été débattue et on trouve les prémisses du consensus actuel de la définition du concept de la température chez Descartes ${ }^{2}$ :

Dans un corps échauffé, sont des particules animées d'un mouvement peu ample, mais très rapide; de ce mouvement, un corps donné, porté à un degré de chaleur déterminé, contient une certaine quantité; cette quantité de mouvement calorifique dans un corps chaud, c'est la quantité de chaleur qu'il renferme.

À l'échelle microscopique, la matière est composée d'atomes, éventuellement assemblés en molécules. Ces particules s'agitent dans tous les sens, se heurtent les unes les autres, rebondissent, repartent dans d'autres directions, en un mouvement chaotique, désordonné : on parle de mouvement brownien, du nom du botaniste Robert Brown, qui en observa les conséquences pour la première fois 3 Pour le physicien, la température est une mesure de l'agitation moyenne de ces particules. Plus les particules bougent vite, plus on dit que la température est élevée. Quand on dit que l'air de la pièce est à $20^{\circ} \mathrm{C}$, cela veut dire que les molécules qui le composent bougent dans tous les sens à environ 300 mètres par seconde $(\mathrm{m} / \mathrm{s})$. De la même manière, les molécules qui composent un solide ou un liquide bougent en permanence plus ou moins librement, vibrant parfois simplement sur place; la force de leur agitation est mesurée par la température.

Cette image de la température est extrêmement riche et met en relief deux idées importantes. Tout d'abord, elle implique qu'il existe une température minimale. Un corps est d'autant plus froid que ses particules bougent peu; la température la plus basse est donc obtenue lorsque toutes les particules sont immobiles. En effet, comment imaginer ralentir davantage des particules immobiles? La température qui correspond à l'immobilité de toutes les particules s'appelle le zéro absolu; elle a été estimée pour la première fois vers 1702 par Guillaume Amontons ${ }^{4}$, et on sait aujourd'hui qu'elle vaut $-273,15^{\circ} \mathrm{C}$. C'est une limite absolue, et on a coutume de s'en servir comme référence : on compte les températures à partir du zéro absolu et, au lieu d'employer les degrés Celsius $\left({ }^{\circ} \mathrm{C}\right)$, on parle de kelvins $(\mathrm{K})$ (les kelvins ne sont pas des degrés, car ils constituent une mesure absolue de la température du corps et non une mesure relative.) Comptée en kelvins, une température quatre fois plus élevée correspond à des particules bougeant deux fois plus vite.

Ensuite, cette image de la température permet également de comprendre comment le froid permet l'émergence de structures. À courte distance, deux particules peuvent interagir et avoir tendance à s'accrocher l'un à l'autre. Cependant, si les particules bougent trop vite et dans tous les sens, leur agitation risque de les empêcher de s'associer et donc de former des édifices complexes. Cette compétition entre l'agitation désordonnée de la température et les interactions entre particules est un élément central pour comprendre les effets du froid.

2. René Descartes, cité par Pierre Duhem, L'évolution de la mécanique et autres textes, Paris, Vrin, Mathesis, 1992, p. 7.

3. Robert Brown, "A brief account of microscopical observations made in the months of June, July and August, 1827, on the particles contained in the pollen of plants ; and on the general existence of active molecules in organic and inorganic bodies», Philosophical Magazine, vol. 4,1828 , p. 161-173.

4. Guillaume Amontons, cité par Kurt Mendelssohn, La recherche du Zéro absolu, Paris, Hachette, coll. «L'univers des connaissances», 1966, p. 11 

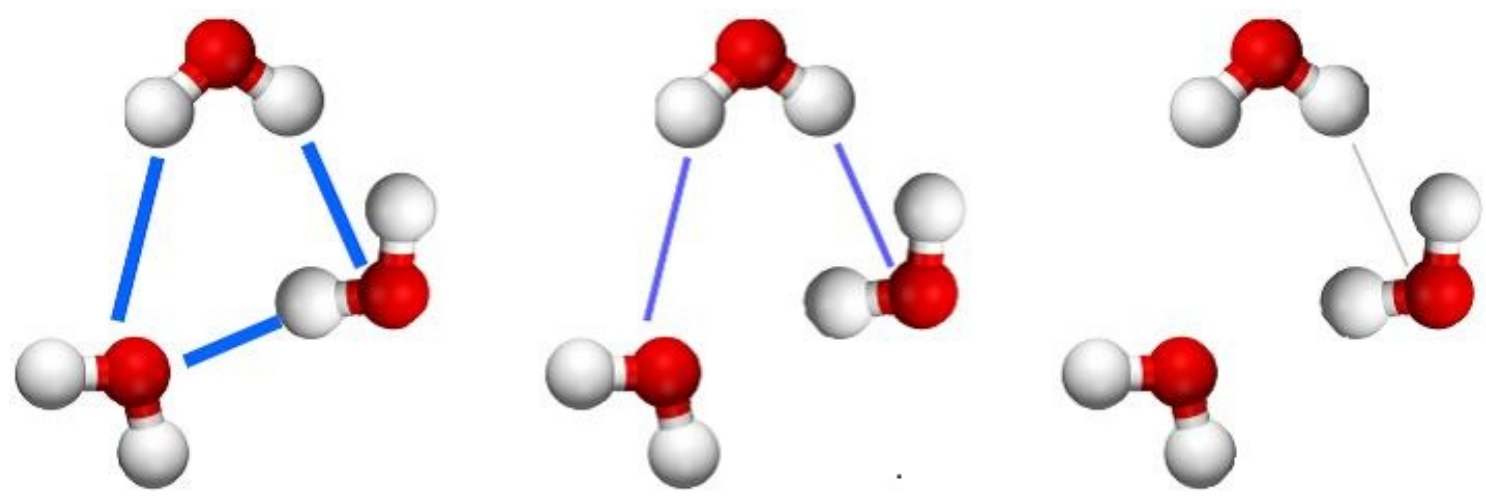

FIGURE 1 - Les états de l'eau. Qu'elle soit solide (à gauche), liquide (au centre) ou gazeuse (à droite), l'eau est toujours composée des mêmes molécules. La différence entre les états de la matière vient des liaisons qui rassemblent ces molécules. Source : Sauf indication contraire, toutes les figures sont de l'auteur.

On peut illustrer cette idée par un exemple bien connu : les états de la matière, et en particulier de l'eau. L'eau liquide est composée de molécules d'eau, $\mathrm{H}_{2} \mathrm{O}$, elles-mêmes constituées de deux atomes d'hydrogène et d'un atome d'oxygène. Sous forme de vapeur, de liquide ou de solide, la formule de l'eau est toujours la même : $\mathrm{H}_{2} \mathrm{O}$. Pourtant, les comportements des trois formes sont radicalement différents. La différence tient à la présence de liaisons entre les molécules d'eau (figure 1). À l'état solide, les molécules sont fermement attachées les unes aux autres par des liaisons solides, qui assurent la cohésion et la rigidité de la glace. Si les molécules bougent trop vite, une partie de ces liaisons est brisée : l'eau perd en rigidité et devient liquide. Si toutes les liaisons entre les molécules sont brisées (ou presque), les molécules peuvent s'éparpiller dans tous les sens et on parle de vapeur. On voit ici comment la compétition entre la température, c'est-à-dire l'agitation des molécules, et l'ordre issu des liaisons permet d'interpréter les états de la matière.

Ce raisonnement peut-il s'appliquer à tous les corps? Tous les gaz peuvent-ils devenir liquides, si leur température, ou leur agitation, est suffisamment faible pour que les molécules s'accrochent un peu les unes aux autres? Ou existe-t-il des gaz permanents, incapables de devenir liquides? Cette question a motivé des générations de physiciens qui ont ouvert la marche vers les températures les plus basses 5 .

En 1784, Gaspard Monge devient le premier physicien à liquéfier artificiellement un gaz, en refroidissant du dioxyde du soufre à $-8^{\circ} \mathrm{C}$. Pendant un siècle, les physiciens s'attaquent aux éléments de la classification périodique les uns après les autres, descendant toujours plus bas dans les températures. En 1877, Pictet et Cailletet liquéfient tous deux $1^{\prime}$ oxygène à $-182^{\circ} \mathrm{C}$, soit $77 \mathrm{~K}$. Quelques années plus tard, Olszewski et Wroblewski parviennent à attendre $-196^{\circ} \mathrm{C}$ et à liquéfier l'azote. Ces résultats répondent à la question soulevée un siècle plus tôt par Lavoisier. En effet, l'air que nous respirons est composé à $20 \%$ d'oxygène et à $80 \%$ d'azote. Si la Terre se trouvait portée dans des régions très froides, l'air deviendrait bel et bien liquide et ressemblerait à un mélange d'oxygène et d'azote liquide.

On peut facilement trouver de nombreuses vidéos sur Internet mettant en évidence les propriétés surprenantes de l'air liquide. Ce liquide est incolore et bout au contact de l'air. Proche de $-200^{\circ} \mathrm{C}$, il gèle immédiatement n'importe quel objet immergé et transforme l'humidité ambiante en cristaux de glace. Versé sur une surface plane, il forme des gouttelettes qui flottent sans frottement et glissent rapidement.

À la fin du XIXe siècle, tous les gaz sont liquéfiés. Tous, vraiment? Non, car l'hélium, particulièrement récalcitrant, résiste encore et toujours à la communauté scientifique. Pendant quelques années, on envisage même que l'hélium soit le seul gaz permanent de l'Univers, condamné à rester gazeux même aux températures les plus basses. Coup de tonnerre en 1908: dans son laboratoire de Leide, Kammerlingh Onnes mène un dernier assaut et, en atteignant la température de $4 \mathrm{~K}$, parvient à liquéfier l'hélium et à clore ainsi la première partie de la course vers les basses températures.

5. Robert Locqueneux, Histoire de la thermodynamique classique : de Sadi Carnot à Gibbs, Paris, Belin, coll. «Science», 2009. 


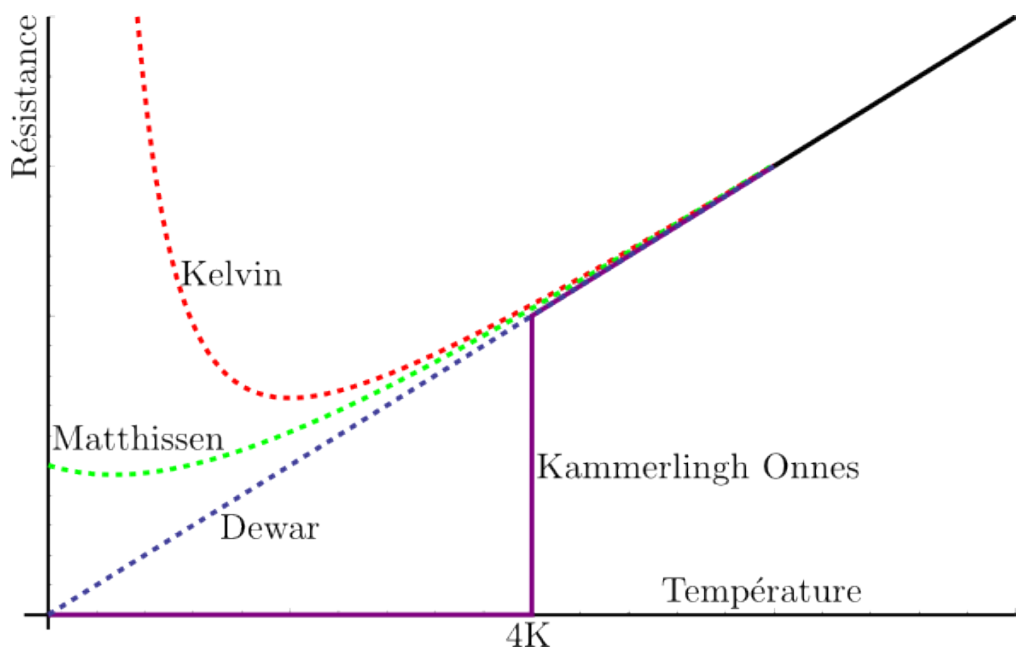

FIGURE 2 - Résistance du mercure en fonction de la température. Les trois courbes en pointillé sont des modèles théoriques, la courbe pleine décrit le résultat expérimental obtenu par Kammerlingh Onnes en 1911.

\section{De $-269^{\circ} \mathrm{C}$ à $-273^{\circ} \mathrm{C}$. La supraconductivité}

La quête des basses températures n'en est pas restée là. Si le froid peut changer tous les gaz en liquides, il génère de nombreux autres changements que les physiciens, curieux et armés d'hélium liquide à $-269^{\circ} \mathrm{C}$, voulaient explorer.

Après son succès dans la liquéfaction de l'hélium, Kammerlingh Onnes s'est tourné vers un second problème : comment les métaux conduisent-ils le courant électrique lorsqu'ils sont refroidis? Des expériences précédentes avaient montré que la résistance électrique diminuait avec la température : un métal plus froid conduit mieux l'électricité qu'un métal plus chaud. Mais au début du XXe siècle, plusieurs théories s'affrontaient pour extrapoler ce comportement jusqu'au plus près du zéro absolu. Certains (dont Lord Dewar ${ }^{6}$ ) pensaient que la résistance continuerait à tendre vers zéro et qu'un métal totalement refroidi pourrait conduire le courant sans aucune perte (figure 2, courbe bleue). D'autres (dont Lord Kelvin 7 remarquaient que le courant électrique correspond au mouvement des électrons, qui courent dans le métal. Si le zéro absolu signifie l'immobilité parfaite, alors les électrons eux-mêmes doivent être figés et donc incapables de porter le courant : la résistance doit donc augmenter brutalement à très basse température (figure 2, courbe rouge). D'autres enfin (dont Matthissen 8 , envisageaient une solution intermédiaire qui verrait la résistance électrique tendre vers une valeur finie et non nulle (figure 2, courbe verte).

Kammerlingh Onnes mène l'expérience en refroidissant du mercure par de l'hélium liquide. Son résultat est complètement inattendu : la résistance du mercure tombe brutalement à zéro quand la température atteint 4,2K (figure 2, courbe violette)! Tout à coup, le mercure conduit le courant sans aucune perte : on dit qu'il est devenu supraconducteur. À l'heure actuelle, on estime qu'un courant électrique peut tourner en boucle dans un anneau supraconducteur pendant plus de 100000 ans sans que la moindre perte puisse être mesurée. Il faut également noter que cette transition vers la supraconductivité est brutale : tout comme l'eau est liquide juste au-dessus de $0^{\circ} \mathrm{C}$ et solide juste au-dessous, avec des propriétés radicalement différentes, le mercure présente une résistance électrique non nulle juste au-dessus de 4,2K et absolument aucune résistance juste au-dessous. Cette discontinuité des propriétés physiques est caractéristique des changements d'état. On voit ici que le froid a des effets autres que le passage solide/liquide/gaz : il peut aussi induire une transition supra/normale.

Un domaine de recherche particulièrement actif vise à produire un matériau supraconducteur à «haute température critique», c'est-à-dire qui deviendrait supraconducteur au-dessous d'une température relativement élevée (et en tout cas plus facilement atteignable que quelques kelvins). À l'heure actuelle, les matériaux les plus performants deviennent supraconducteurs dès qu'ils atteignent $-135^{\circ} \mathrm{C}$. Cependant, ces matériaux sont le plus souvent des céramiques, friables et cassantes, dont les propriétés mécaniques limitent considéra-

\footnotetext{
6. Stephen Blundell, La supraconductivité, 100 ans après, Paris, Belin, coll. «Pour la science», 2011, p. 32.

7. Ibid.

8. Ibid.
} 


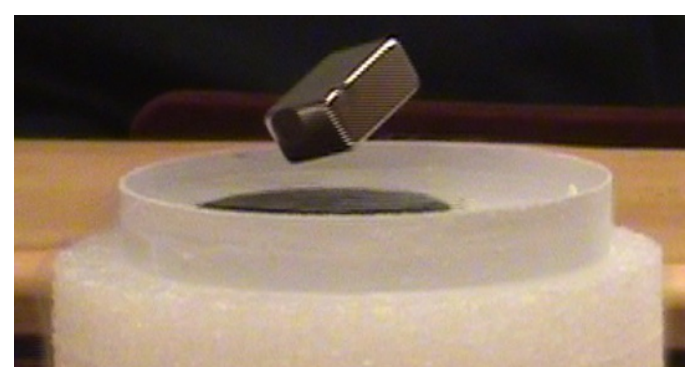

FIGURE 3 - La lévitation magnétique. La pastille noire posée dans la coupole est un supraconducteur refroidi par de l'azote liquide. Un aimant est approché : il est repoussé par la pastille et flotte à quelques millimètres au-dessus du support.

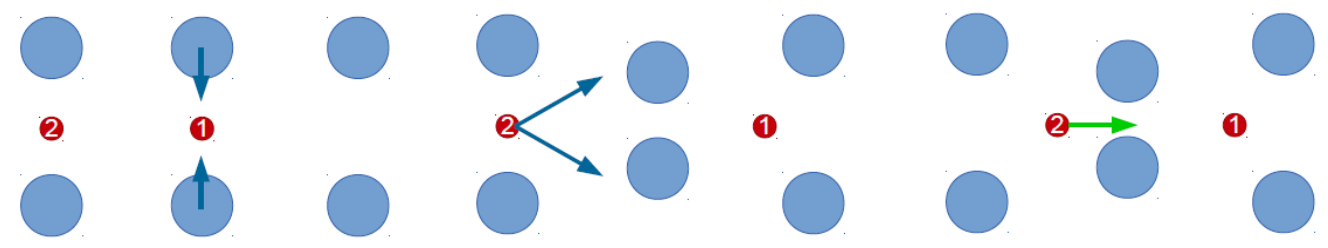

FIGURE 4 - Théorie BCS : comment deux électrons peuvent-ils s'attirer?

blement l'usage industriel.

Une autre conséquence surprenante de la supraconductivité a été découverte par Walther Meissner et Robert Ochsenfeld en 1933 : un supraconducteur repousse les champs magnétiques. Cet effet Meissner est à l'origine de la lévitation magnétique, l'une des manifestations les plus spectaculaires de la supraconductivité. À température ambiante, une pastille supraconductrice n'a aucune propriété magnétique remarquable et on peut en approcher un aimant sans effort. Mais si la pastille est refroidie au-dessous de sa température critique, elle devient supraconductrice et repousse les aimants qui s'en approchent. On peut alors faire voler un aimant au-dessus de la pastille (figure 3); cet effet est suffisamment fort pour qu'une équipe du laboratoire Matériaux et Phénomènes Quantiques construise un MagSurf 9 , un skate supraconducteur digne du film Retour vers le futur qui flotte à quelques centimètres sur un rail d'aimants!

Entre la découverte de ces phénomènes et leur modélisation théorique, il se passe plus de quarante ans. Le premier modèle complet de la supraconductivité naît en 1957 de la collaboration de trois physiciens : John Bardeen, Léon Cooper et John Robert Schrieffer. Dans la théorie BCS, les propriétés des supraconducteurs viennent de la capacité des électrons dans le métal à former des paires qui présentent un comportement collectif. Une analogie classique compare les électrons à des poissons dans l'eau. À température ambiante, les électrons se déplacent sans tenir compte les uns des autres, comme un nuage de poissons désordonné. Audessous de la température critique, les électrons s'apparient et se comportent comme un banc de poissons, dans lequel chaque individu ajuste se trajectoire en fonction des autres. Impossible de venir déranger un poisson sans déranger tout le banc! De la même manière, dans un métal devenu supraconducteur, il est impossible d'empêcher la propagation d'un électron sans perturber tous les autres. Il devient alors énergétiquement coûteux de s'opposer au courant électrique, et les électrons-poissons peuvent donc se déplacer sans encombre, ce qui se traduit par la disparition de toute dissipation.

Il peut sembler surprenant que deux électrons puissent former une paire : tous les électrons sont chargés négativement et par conséquent se repoussent les uns les autres. Comment pourraient-ils s'attirer au point de s'apparier? L'explication tient à la présence du réseau atomique dans lequel évoluent les électrons (figure 4) : lors de son déplacement dans le réseau, un électron attire (très légèrement) vers lui les atomes environnants. Par conséquent, un deuxième électron verra dans le sillage du premier des atomes légèrement plus rapprochés et sera donc attiré dans cette direction préférentiellement. Tout se passe comme si le premier électron avait attiré le second à sa suite!

9. Daniel Suchet, La magie renversante des supraconducteurs] 2013 [Disponible sur : http ://www.actusf.com/spip/L-actu-des-sciencesFevrier-2013.html] 

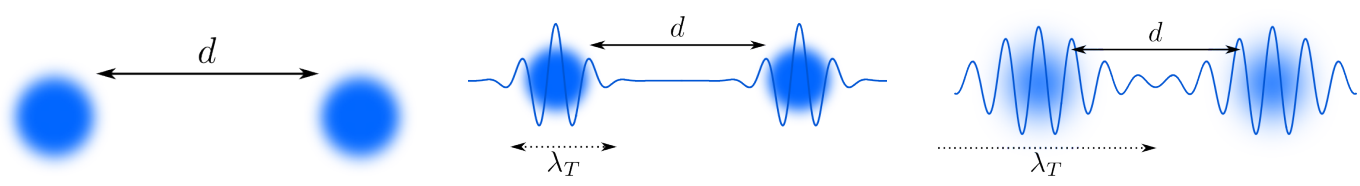

FIGURE 5 - Les températures ultrabasses. Considérons deux particules, distantes de $d$. Chacune de ces particules est décrite par une onde, qui présente une taille $\lambda_{T}$. Si $\lambda_{T}$ est plus grand que $d$, les ondes se superposent les unes aux autres et la nature quantique du système devient prédominante.

Cette image permet de comprendre pourquoi un supraconducteur ne peut exister qu'à basse température. À température ambiante, les atomes vibrent dans tous les sens et le déplacement dû au passage d'un électron est noyé dans l'agitation moyenne. Il faut diminuer considérablement la température (et donc l'agitation) pour que le faible effet des électrons sur les atomes ne soit pas complètement négligeable. Cependant, comme toutes les images, cette description du phénomène reste limitée : elle n'explique ni pourquoi l'attraction entre électrons engendre l'apparition de paires (et pas de triplets, etc.) ni pourquoi les paires d'électrons présentent un comportement collectif si particulier. Pour décrire correctement le phénomène, il faudrait utiliser une théorie physique plus subtile : la mécanique quantique, qui s'avère indispensable pour comprendre ce qui se passe lorsqu'on descend encore plus proche du zéro absolu.

\section{Vers le zéro absolu. Les atomes ultrafroids}

Les techniques de cryogénie ont considérablement progressé depuis l'époque de Kammerlingh Onnes. À l'heure actuelle, les meilleurs cryostats sont capables de descendre jusqu'à quelques millikelvins, c'est-à-dire quelques millièmes de degré au-dessus du zéro absolu. Ces températures sont remarquables : les températures dont nous parlons ici sont cent mille fois plus basses que les 300 kelvins de la température ambiante! Par comparaison, rappelons que la surface du Soleil, portée à quelque $5000 \mathrm{~K}$, n'est qu'environ dix fois plus chaude que la température ambiante. C'est à des températures encore bien inférieures que nous allons descendre pour achever notre plongée.

Particulièrement actif depuis une vingtaine d'années, le domaine des atomes froids s'intéresse à des températures dites «ultrabasses», où la nature quantique des particules, déjà mentionnée dans la supraconductivité, devient omniprésente.

À quel point doit-on être froid pour être ultrafroid et d'où viennent ces effets quantiques? Les ordres de grandeur apparaissent à l'échelle microscopique (figure 5). Pour décrire un nuage de gaz, une première longueur caractéristique semble évidente : la distance moyenne entre les particules, qu'on peut noter $d$. Mais cette longueur ne suffit pas : d'après la mécanique quantique, les particules ne sont pas ponctuelles, mais sont décrites par une onde qui s'étend dans un certain volume. L'extension de ce paquet d'onde est donnée par la longueur d'onde de de Broglie, notée $\lambda_{T}$, qui dépend de la masse des particules et de la température.

Pour un gaz à température ambiante (l'air par exemple), la distance entre atomes $d$ est beaucoup plus grande que la longueur d'onde de de Broglie; et chaque petit paquet d'onde est bien isolé des autres. Mais si la température baisse, longueur d'onde de de Broglie augmente; elle peut devenir plus grande que la distance qui sépare les particules si la température est assez basse. Les paquets finissent alors par se superposer et les particules interfèrent les unes avec les autres. On parle dans ce cas de températures ultrabasses. Pour un gaz d'atomes, il faut atteindre des températures de l'ordre de 100 nanokelvins (100 milliardièmes de degré au-dessus du 0 absolu) pour entrer dans ce régime.

Lorsque les paquets d'onde se superposent, les particules ne peuvent plus être isolées les unes des autres, mais présentent au contraire un comportement collectif. On observe alors deux types de comportements : certaines particules, appelées bosons, ont tendance à toutes s'accumuler dans la même configuration et forment un nouvel état de la matière appelé condensat de Bose- Einstein. À l'inverse, d'autres particules, les fermions, refusent catégoriquement d'être deux dans la même configuration et chaque nouvelle particule doit trouver une configuration non occupée. C'est dans cette seconde catégorie que se rangent entre autres les électrons. La première réalisation expérimentale d'un condensat de Bose-Einstein en 1995 par l'équipe d'Eric Cornell et Carl 


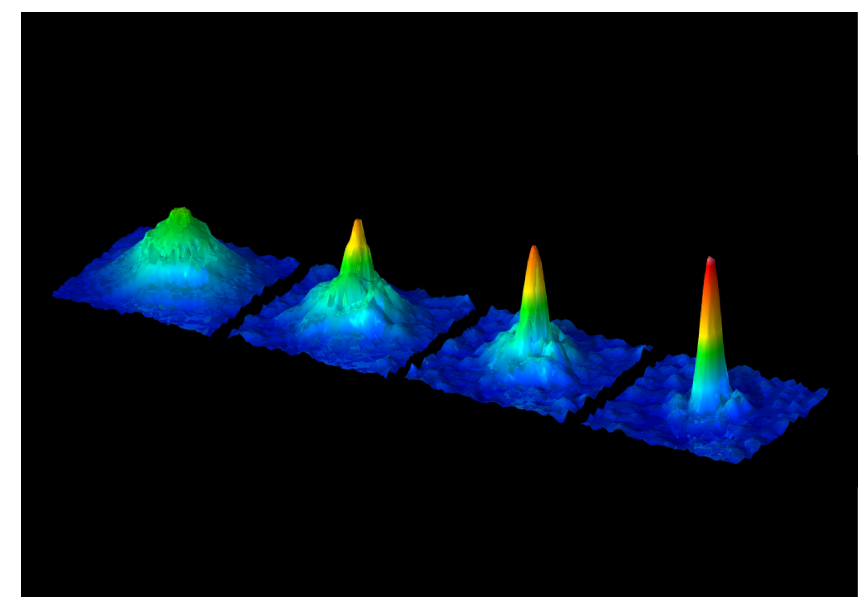

FIGURE 6 - Formation d'un condensat de Bose-Einstein. Chacune des images présente un nuage d'environ six milliards d'atomes de Rubidium 87, qui se comporte comme un boson. La seule différence entre les nuages est leur température (de gauche à droite : $800 \mathrm{nK}, 400 \mathrm{nK}, 200 \mathrm{nK}$ et 100nK). Au fur et à mesure que la température diminue, on voit les atomes s'accumuler dans une seule distribution, qui devient de plus en plus piquée. Source : Équipe BEC, Max Planck Institute of Quantum Optics, 2003.

Wieman (figure 6) montre la vivacité du domaine.

L'un des enjeux majeurs de ce domaine de recherche a été imaginé par l'idole des physiciens, Richard Feynman, qui évoque en 1982 un simulateur quantique universel ${ }^{10}$, une machine susceptible d'être ajustée pour simuler n'importe quel système physique qu'on souhaite étudier. Le monde réel est horriblement compliqué, et le nombre de facteurs intervenant dans un problème est quasiment infini. Pour élaborer une théorie, le physicien ne retient que quelquesuns de ces paramètres et néglige les autres; il établit ainsi un modèle simplifié qu'il espère néanmoins fidèle à la réalité. Pour tester sa théorie, le physicien doit s'assurer que le système décrit par son modèle simplifié se comporte effectivement comme le système réel qu'il veut décrire. Pour ce faire, il peut calculer certaines propriétés à partir de sa théorie puis comparer ses prédictions aux réactions réelles du système : réagit-il comme prévu à tel ou tel stimuli? Se déforme-t-il comme l'affirment les calculs? Malheureusement, dans la plupart des cas, les calculs sont complexes au point d'être irréalisables, même par ordinateur. C'est là que l'idée de Feynman prend toute sa force : au lieu de mener ces calculs, on peut simuler les propriétés du modèle en réglant la machine pour qu'elle suive exactement la théorie. Si le comportement du simulateur ressemble au comportement du système réel, c'est que la théorie décrit correctement son objet d'étude; à l'inverse, si le simulateur ne se comporte pas du tout comme le système réel, c'est qu'il manque dans la théorie des éléments cruciaux.

Les atomes ultrafroids se prêtent particulièrement bien à ce projet. En effet, ces atomes forment un système particulièrement ajustable : on peut non seulement dessiner le paysage dans lequel ils évoluent avec des faisceaux laser, mais également ajuster leurs interactions avec un champ magnétique et les forcer ainsi à s'attirer ou à se repousser les uns les autres, plus ou moins fortement.

On peut ainsi reproduire des comportements analogues à ceux des supraconducteurs (figure 7) : un nuage d'atomes fermioniques joue le rôle des électrons dans le métal et un réseau optique reproduit le potentiel périodique que les électrons ressentent habituellement. Les interactions entre atomes sont réglées de façon à ce que deux atomes s'attirent légèrement - comme les électrons de la théorie BCS. On observe alors un comportement identique à celui des supraconducteurs, mais dans un système parfaitement manipulable où on peut varier chacun des paramètres pour étudier son influence sur l'ensemble du système.

Pour le physicien, le froid n'est pas tant un produit qu'une force de production. De la cristallisation de la glace à la formation des condensats de Bose-Einstein en passant par l'appariement des électrons supraconducteurs, la diminution progressive de l'agitation thermique permet l'émergence de structures de plus en

10. Richard Feynman, «Simulating physics with computers», International Journal of Theoretical Physics, vol. 21, 1982. 

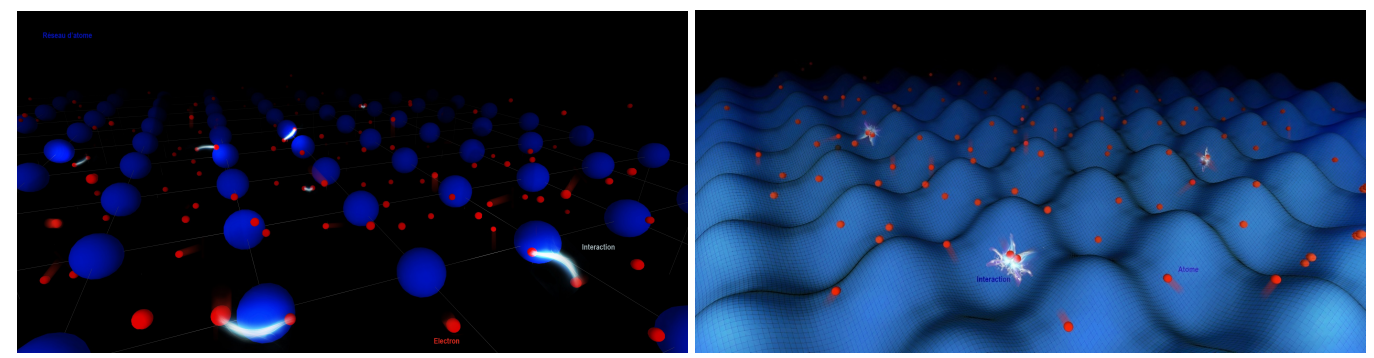

Figure 7 - Vues d'artiste par Germain Morisseau. Dans un métal, les électrons (en rouge) évoluent dans un réseau périodique d'ions (en bleu) et interagissent entre eux (à gauche). De manière analogue, on peut placer des atomes (en rouge) dans un réseau optique (en bleu) et ajuster leurs interactions pour qu'elles soient identiques à celles des électrons (à droite). Source : Germain Morisseau.

plus subtiles. L'exploration des basses températures constitue donc une aventure à la fois technique (comment parvenir à atteindre des températures de plus en plus basses?) et fondamentale (que se passe-t-il quand on atteint effectivement ces températures?). Si les questions soulevées et les méthodes employées ont beaucoup évolué depuis le XVIIIe siècle, ce domaine de recherche reste à l'heure actuelle particulièrement actif, et les expériences d'atomes ultrafroids montrent que, à l'extrême limite du zéro absolu, c'est l'universalité même de la physique que le froid fait émerger.

Pour une visite virtuelle d'un laboratoire d'atomes froids, voir l'histoire courte réalisée par Anne Papillault et Jean-François Dars 11.

Ce texte a été présenté le 13 Décembre 2013 lors du Colloque International Pluridisciplinaire Le Froid, adaptation, production, représentations, effets co-organisé par le laboratoire de recherche CEARC (cultures, environnements actique, représentation climat) de l'observatoire de Versailles Saint Quentin (OVSQ) et le laboratoire international d'étude multidisciplinaire comparée des représentations du Nord de l'Université du Québec à Montréal.

11. Daniel Suchet, Jeux combinatoires, réalisé par Anne Papillault et Jean-François Dars [Disponible sur : http :/ / histoires-courtes.fr /\#page=Suchet] 